\title{
Single particle electron microscopy
}

\author{
Egbert J. Boekema $\cdot$ Mihaela Folea • \\ Roman Kouřil
}

Received: 13 November 2008/Accepted: 19 May 2009/Published online: 10 June 2009

(c) The Author(s) 2009. This article is published with open access at Springerlink.com

\begin{abstract}
Electron microscopy (EM) in combination with image analysis is a powerful technique to study protein structures at low, medium, and high resolution. Since electron micrographs of biological objects are very noisy, improvement of the signal-to-noise ratio by image processing is an integral part of EM, and this is performed by averaging large numbers of individual projections. Averaging procedures can be divided into crystallographic and non-crystallographic methods. The crystallographic averaging method, based on two-dimensional (2D) crystals of (membrane) proteins, yielded in solving atomic protein structures in the last century. More recently, single particle analysis could be extended to solve atomic structures as well. It is a suitable method for large proteins, viruses, and proteins that are difficult to crystallize. Because it is also a fast method to reveal the low-to-medium resolution structures, the impact of its application is growing rapidly. Technical aspects, results, and possibilities are presented.
\end{abstract}

Keywords Electron microscopy $\cdot$ Single particle analysis · Photosynthesis

\section{Introduction}

Direct information about the three-dimensional (3D) structure of a protein complex is essential for understanding its functional organization. At present, electron microscopy (EM) is a widely applied technique for studying the structure of proteins and membranes, but it is still less common than

E. J. Boekema $(\bowtie) \cdot$ M. Folea $\cdot$ R. Kouřil

Biophysical Chemistry, University of Groningen, Nijenborgh 4, 9747 AG Groningen, The Netherlands

e-mail: e.j.boekema@rug.nl
X-ray diffraction where solving the 3D structure of proteins became almost routine, once suitable crystals have been obtained. On the other hand, X-ray diffraction has two disadvantages in comparison to EM. First, the main disadvantage is the problem of getting well-ordered, large enough crystals. The interaction of electrons with material is stronger than for X-rays by a factor of about 10,000 . This makes EM a useful technique for imaging single-layer 2D crystals or single protein molecules on a thin support film, in contrast to the thicker specimens in the (sub) micron range, used in $\mathrm{X}$-ray diffraction. A second reason is that only diffraction patterns are obtained, whereas EM results in direct information in the form of images.

Imaging of thin metal foils or gold clusters by EM will easily provide projections with atomic details, but obtaining structures of proteins at high resolution is much harder work. Why? The contrast in the electron microscope is caused by scattering. Biological samples containing mostly light elements give images with low contrast, since the scattering of electrons is proportional to the atomic number Z. Besides, radiation damage by the electron beam can easily destroy biological samples. Radiation damage cannot be avoided, but only minimized (i) by cooling the specimen to either liquid nitrogen or liquid helium temperature and (ii) by minimizing the electron dose. The latter results in noisy electron micrographs with hardly visible biological objects. Therefore, image analysis techniques have been developed to improve the signal recorded in the EM pictures.

In EM image analysis, improving the signal of an object is performed by averaging. By adding hundreds or, if possible, many thousands of projections, the signal improves substantially and trustworthy electron density maps are obtained. There are two general methods for averaging of $2 \mathrm{D}$ projections, depending on the object. One 
method, electron crystallography, is based on filtering images of periodic objects, which are usually 2D crystals. The other, single particle averaging, deals with randomly oriented single molecules. Electron crystallography was able to solve some important membrane protein structures, at a time when only a limited number of such structures were solved by X-ray diffraction. Bacteriorhodopsin (Henderson et al. 1990) and Light-harvesting complex II (LHCII) from pea (Kühlbrandt et al. 1994) were the first proteins to be completed, although more recently slightly better structures have been provided by X-ray diffraction. Electron crystallography needs well-ordered, large 2D crystals. The preferential size is a few micrometers, and such crystals are not always easy to grow. This is clearly a reason why electron crystallography is not a mainstream technique and also why EM is moving toward single particle analysis. Other advantages of single particle EM versus $2 \mathrm{D}$ crystal analysis are the facts that samples of smaller quantities are needed and low purity is possible, at least for determination of 2D projection maps. A good introduction to the technique of $2 \mathrm{D}$ crystal analysis can be found in Yeager et al. (1999).

\section{Specimen preparation: cryo-EM and classical negative staining}

Since modern electron microscopes have enough resolving power for structural studies of macromolecules, factors other than instrumental ones are of equal importance. The specimen preparation method is one of these factors, and it strongly determines the ultimate results that can be achieved. In the negative staining technique, the contrast is enhanced by embedding biomolecules in a heavy metal salt solution (see Harris and Horne 1994 for a review). On drying, the metal salt fills cavities and the space around the molecules, but does not penetrate the hydrophobic protein interior. As a result, negatively stained specimens show protein envelopes with good contrast. As an alternative for negative staining, cryo-EM was developed in the 1980s (Adrian et al. 1984). By rapid cooling of a thin layer of an aqueous solution of macromolecules on an EM grid, a thin amorphous layer of ice is formed, in which objects are visible without any staining agent. Ice-embedded specimens very much reflect cellular aqueous situations, and hence the method quickly became popular within the field. Because the contrast is only caused by the difference in density between amorphous ice $\left(0.93 \mathrm{~g} / \mathrm{cm}^{3}\right)$ and protein $\left(1.3-1.36 \mathrm{~g} / \mathrm{cm}^{3}\right)$, it is rather low in comparison to negative staining.

It is obvious that for large objects such as symmetric virus molecules, cryo-EM is superior to negative staining. However, in the case of unstable protein complexes, which cannot be purified to homogeneity (e.g., large, transient membrane complexes), unstained specimens can be a real problem. Due to the low contrast, the object of choice cannot be discriminated from all kinds of contaminants and breakdown products. The low contrast is, however, likely to be improved in the near future by instrumental improvements, such as implementing phase plates in the microscopes, such as the Zernike phase plate (Yamaguchi et al. 2008).

There are several advantages of cryo-EM of vitrified specimens: specimen flattening and other drying artifacts are circumvented. Moreover, cryo-images better reflect the true density of a protein, because the contrast directly originates from scattering by the protein rather than from the surrounding stain. Also, the interaction of negative stain with the protein is often quite complex if the object is not fully embedded. In thinner stain layers, the upper part of the protein could easily be less well embedded in the stain layer, as pointed out in Fig. 1. This means that the contributions of the upper- and lower half of a protein in the final recorded image do not have the same weighting. In contrast, the embedding in a full ice layer gives a more straightforward signal. Cryo-negative staining represents a

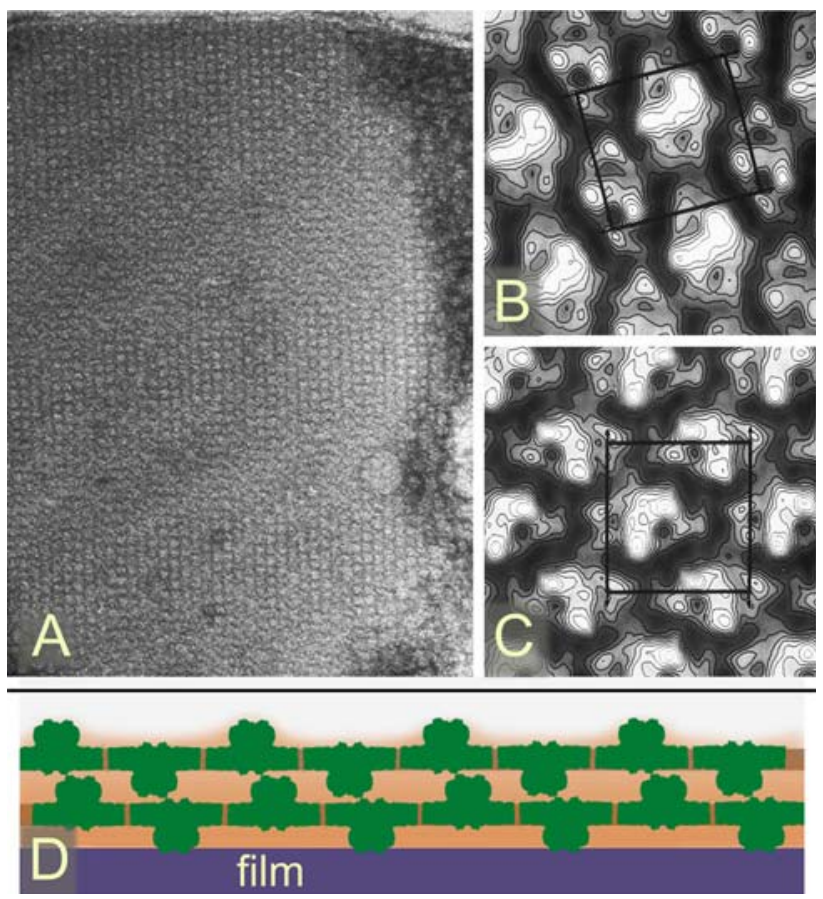

Fig. 1 An example of the footprint effect of negative staining. a A part of a double-layered two-dimensional crystal containing about 1500 photosystem I monomers from a cyanobacterium (Böttcher et al. 1992). b, c Filtered images resulting from a crystallographic analysis in which the two layers could be separated. The crystal is composed of rows of monomers. Within the rows, the monomers are either upor down-oriented, and there is a substantial difference in overall contrast between individual rows of monomers in the upper layer with respect to the lower layer. $\mathbf{d}$ Scheme explaining how the uneven stain density (brown) causes the difference between the two layers, which are identical in protein arrangement 
complementary method for the conventional negative stain $\mathrm{EM}$ and a valuable alternative in particular for situations where cryo-EM reaches its limits in terms of visibility of the protein complexes (De Carlo et al. 2008). In cryonegative staining, particles become embedded in a rather thick layer of stain which is not fully dehydrated, which may prevent flattening and preferential staining.

\section{Single particle EM: the method}

The purpose of single particle EM is to determine the structure of macromolecules from images of individual particles or single particle projections. Because isolated macromolecules, prepared on a carbon support film or in a thin layer of ice over a holey carbon film, usually exhibit a full range of orientations, resulting projections will differ as well, and substantial processing is needed before averaging can take place. Basically, the method of single particle analysis consists of only a few crucial steps, of which two are illustrated in Fig. 2. If projections result from one type of orientation on the support film, averaging is possible after alignment. The alignment step brings projections in equivalent positions by computing rotational and translational shifts. In the case of the example, a supercomplex of trimeric photosystem I (PSI) surrounded by a ring of 18 copies of the antenna protein IsiA, a set of 5000 projections has been brought in register. It can be seen that by increasing the number of summed projections the noise is gradually reduced (Fig. 2, upper part). It is very obvious that from individual, noisy projections the number of IsiA copies cannot be retrieved and that processing is indispensable.

Just summing of projections, however, is meaningless when the projections arise from particles in different orientations toward the plane. In order to deal with this, data sets have to be treated with multivariate statistical analysis together with automated classification (see Van Heel et al. 2000; Frank 2002 for reviews on single particle EM). After statistical analysis and classification, those images that are most similar can be grouped together. The output of the classification is "classes" of groups of homogeneous projections. In the case of the data set of PSI-IsiA, it turned out that further processing of projections after the alignment step improved the final sums. The particle projections were not all identical, because small tilt variations on the support film led to different positions. The statistical analysis and classification showed that only a small number of projections had threefold rotational symmetry, indicative for a position parallel to the membrane (Fig. 2, lower row, left). The other two classes (middle and right) show the supercomplex in tilted positions.

3D reconstructions can be obtained from large sets of projections of objects under different angles. In favorable
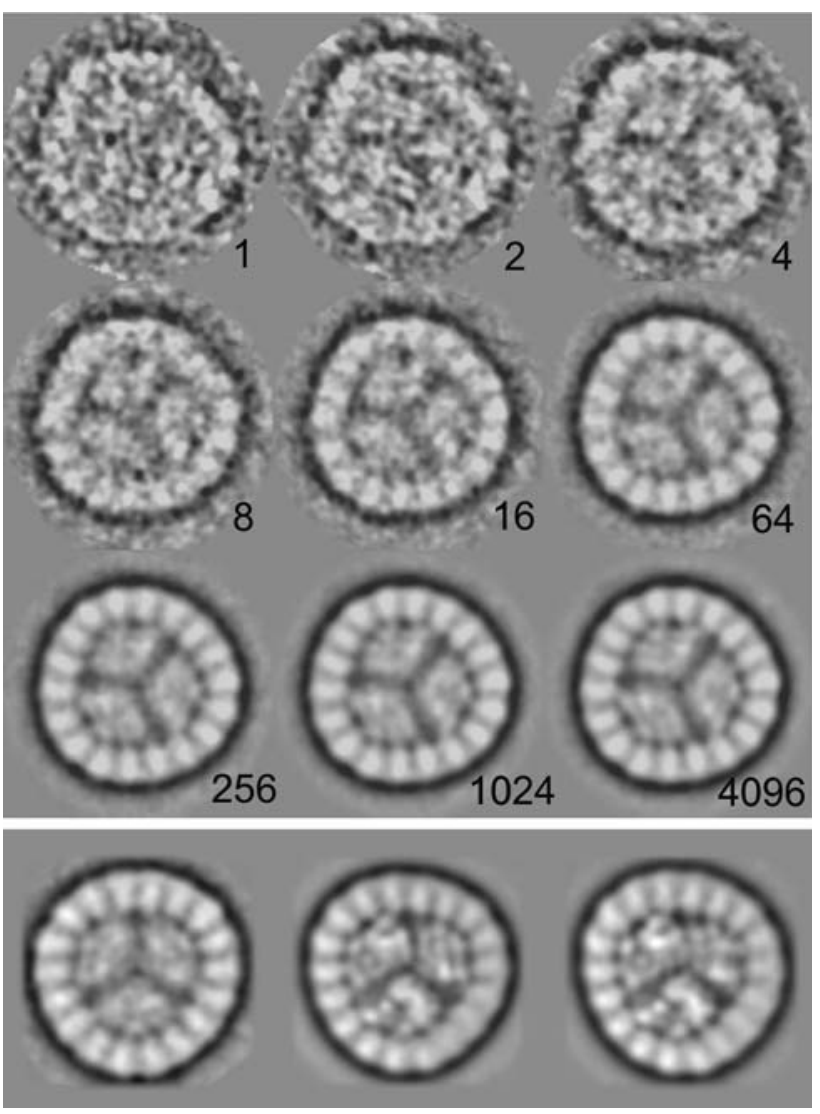

Fig. 2 The basics of single particle EM, explained from an analysis of the photosystem I-IsiA supercomplex from the cyanobacterium Synechococcus 7942, extracted from negatively stained EM specimens (Boekema et al. 2001). After translational and rotational alignment of a data set of about 5000 single particle projections showing the complex in a position as in the membrane plane, sums with increasing numbers of copies in equivalent positions show the gradual improvement in the signal-to-noise ratio (upper part of the picture). However, these particle projections may not all be identical, because small tilt variations on the membrane plane may lead to different positions. Indeed, after multivariate statistical analysis and classification, it became clear that only a small number of projections show threefold rotational symmetry which is indicative for a position parallel to the membrane (lower row, left). The other two classes (middle and right) show the supercomplex in tilted positions

cases, the molecules show random orientation in the ice layer or on the support film. If not, specimens can be tilted in the microscope in order to obtain 2D projection maps of the molecules viewed from different angles. For the PSIIsiA particle, such a 3D reconstruction was produced (Bibby et al. 2001), but it did not show much more details than that were already visible in the 2D maps, because the complex is a rather flat object. However, in general, 3D information is much more valuable especially for spherical objects as ribosomes and virus molecules.

In the 1980s and 1990s, single particle analysis was still a matter of hard labor, including the recording on photographic emulsion, scanning the images by densitometers 
and processing, which was less sophisticated (Fig. 3a). In recent years, single particle method has been developed much in a direction of automation of all steps, i.e., from automated particle collection to iterative improvements of initial 3D reconstructions. The use of scanning slow-scan CCD cameras, which can be programmed to record hundreds of images in a semi-automated way, helped tremendously (Fig. 3b). In the near future, it is expected that direct electron counters with superior recording qualities will replace the $\mathrm{CCD}$ cameras (Faruqi and Henderson 2007) and that further automation will provide structures within hours after sample insertion in the microscope. In addition, much higher contrast of unstained specimens is possible by application of "novel" phase contrast electron microscopy such as the Zernike phase contrast microscopy (Yamaguchi et al. 2008). This is similar to the phase contrast light microscope, for which Frits Zernike was awarded the Nobel prize for physics in 1953. Implementation in commercial electron microscopes will be a logical next step in improving EM methods.

In 2007, a special issue of the journal of structural biology was devoted to software tools in macromolecular electron microscopy (see Carragher et al. 2007). The
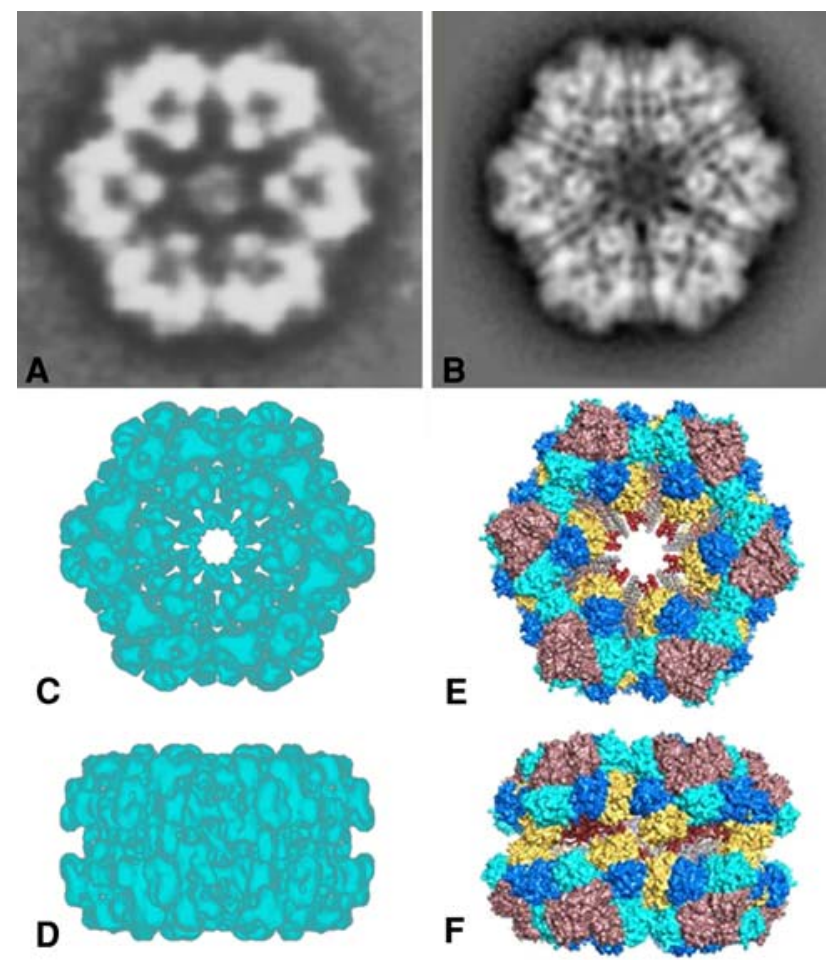

Fig. 3 Example of single particle analysis on a large water-soluble protein, the 180-subunit hemoglobin of the earth worm Lumbricus terrestris. a (Boekema and van Heel 1989). b Sum of 1024 particles at $11 \AA$ resolution in negative stain (R. Kouril unpublished). c, d Two views of a $3 \mathrm{D}$ reconstruction at $13 \AA$ resolution (W. Keegstra and G.T. Oostergetel, unpublished). e, f Model of the high-resolution (3.5 ̊) X-ray structure (Royer et al. 2006) applications described in this issue represent a wide range and variety of software solutions including half a dozen general software packages, such as EMAN and SPIDER, which are popular in the field of single particle analysis. An extensive list of software tools can be found in Wikipedia: http://en.wikipedia.org/wiki/Software_tools_for_molecular_ microscopy.

\section{Resolution in single particle analysis}

In theory, it is possible to obtain high-resolution structures for proteins as small as about 100,000 Da (Henderson 1995). At present, high-resolution is feasible with large, stable water-soluble protein complexes. It has been suggested that over a million particles are necessary for solving to high-resolution a non-symmetric object, although this has not yet been performed. With highly symmetric particles such a resolution has already been obtained. The first protein solved at atomic resolution was a viral protein in the rotavirus DLP (Zhang et al. 2008). Analysis was achieved with only 8,400 particle projections, because by imposing symmetry the densities of 6.6 million protein copies could be used. A lower-symmetrical protein, GroEL, was reconstructed to about $4 \AA$ by making use of internal sevenfold symmetry (Ludtke et al. 2008). At this level of resolution, the $\mathrm{C} \alpha$ amino acid backbone could be traced directly from a cryo-EM reconstruction. For a number of objects medium resolution (just below $10 \AA$ ) has been achieved, enabling the assignment of secondary structure elements, such as $\alpha$-helices.

One good argument in favor of cryo-EM is the resolution, which is better than for negative staining and one of the main drawbacks is the low contrast which leads to a rather limited visibility of the particles in cryo-EM pictures. A nuclear ribonucleoprotein particle (snRNP) of $240 \mathrm{kDa}$ was determined to $10 \AA$ and represents one of the smallest particles determined without any contrasting agent, close to the limit of the technique (Stark et al. 2001).

Because of its high contrast, negative staining is not yet outdated. Results on catalase crystals established that negative staining preserves structural information into the high-resolution range of $4.0 \AA$ (Massower et al. 2001), in contrast the widely accepted current belief that this methodology usually can give a resolution limited to only 20-25 A. On the other hand, it should also be stated that on the same catalase crystals a better resolution of $2.8 \AA$ was obtained in ice. In $2 \mathrm{D}$ maps or $3 \mathrm{D}$ reconstructions a resolution of $8-9 \AA$ by negative staining is possible. Cryo-negative staining structures below $10 \AA$ were obtained from the multiprotein splicing factor SF3b (Golas et al. 2003) and GroEL (De Carlo et al. 2008). For rigid, well-stained molecules, such as worm hemoglobin, our test 
object, a resolution of $11 \AA$ can be achieved in 2D maps from only 1000 summed projections (Fig. 3b). In summary, atomic resolution is possible, but we also have to realize that not all protein complexes are ideal objects for highresolution (see next sections). A drawback of membraneproteins is the fact that they only stay monodisperse in solution within a non-ordered detergent layer (Boekema 1991), which makes projections fuzzy at the circumference.

\section{State of the art in single particle EM}

At present, single particle EM has its highest impact in large multi-subunit structures that cannot be crystallized easily, either in 3D (X-ray crystallography) or 2D (electron crystallography). In the field of photosynthesis, 2D maps of photosynthetic membrane proteins are very helpful in analysis of the peripheral antenna complexes (reviewed in Dekker and Boekema 2005), although many complexes have not yet been analyzed below 10-15 $\AA$. Nevertheless, there is yet a very useful application at medium resolution, which is the combination of EM and X-ray structures. Over the last decade, docking of atomic resolution X-ray structures into the molecular envelopes derived by cryo-EM became popular (reviewed by Unger 2001 and Stahlberg and Walz 2008). At a resolution of about $15 \AA$, pseudoatomic structures can be derived that tell about the interactions on the level of $\alpha$-helices of specific subunits (Heinemeyer et al. 2007); a higher resolution (10 $\AA$ or slightly better) is necessary to predict interaction at the atomic level.

The use of rapid freezing devices in cryo-EM enables to study structural changes within the millisecond range in protein complex during catalysis. The ribosome is probably the best studied example of conformational changes studied by single particle EM (Mitra and Frank 2006). Another example of the hybrid X-ray-EM approach is the worm hemoglobin, already presented earlier. It was crystallized more than 60 years ago, at a time when crystallization was just a method to purify a protein! However, to solve such a large structure from X-ray diffraction patterns, phases need to be generated. The phase problem in structure determination by X-ray diffraction was solved by taking information from a low-resolution 3D model by EM, similar to the one presented in Fig. 3c, d, and this finally helped to solve the structure to atomic resolution (Fig. 3e, f) (Royer et al. 2006).

Because EM has the unique property to see individual molecules, it has another almost non-explored possibility: to work with partly purified proteins, or even non-purified particles from solubilized membranes (the possibility to work on non-purified proteins will be discussed in the last section). In order to correlate structures to specific proteins, however, biochemical techniques and mass spectrometry analysis are needed for final assignment (Arteni et al. 2005). This type of application is still at its infancy, but no doubt, the combination of mass spectrometry and EM will provide us with structural insight on the level of membranes and cellular complexity.

\section{Flexibility of large protein complexes}

It appears that many protein complexes were designed to be flexible to perform their role in the many dynamic processes in life. It is possible to get an impression about the flexibility of multi-subunit complexes by single particle image analysis. This is illustrated by examples of investigations of PSI-IsiA complexes that are formed in cyanobacteria as a response to stress conditions (Fig. 4). We noticed that relatively little detail is resolved in projection maps of some specific PSI-IsiA particles, despite the large numbers of processed projections (Yeremenko et al. 2004; Kouřil et al. 2005a). PSI-IsiA supercomplexes composed trimeric PSI and a single ring of IsiA are well-defined structures (Fig. 4a), whereas some of the monomeric PSI and double rings of IsiA are flexible. For complexes with two complete rings of 14 and 21 IsiA copies, the full structure could not be well resolved, because the monomer and inner ring appear fuzzy (Fig. 4b). The features of the inner ring could be improved by masking the outer ring of the individual projections during an additional alignment step (Fig. 4c). This improvement is at the cost of detail in the outer ring, which demonstrates that the fuzziness in Fig. 4b, c is caused by rotational flexibility between both
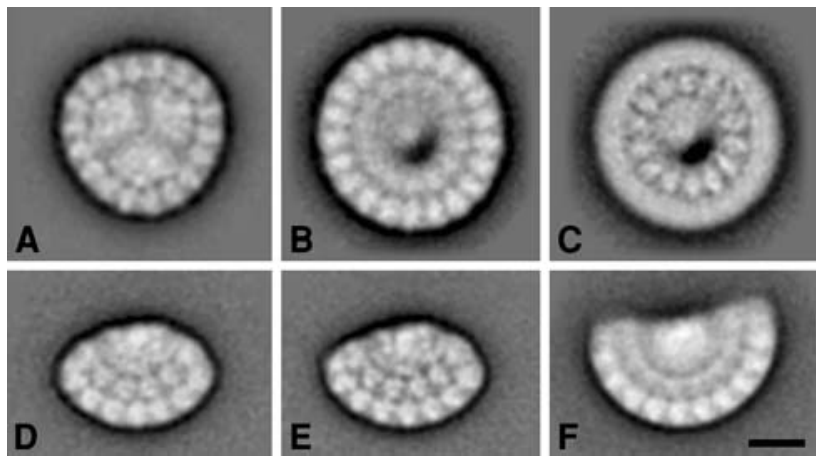

Fig. 4 Supercomplexes of photosystem I-IsiA (PSI-IsiA) with variable amount of flexibility. a The supercomplex consisting of trimeric PSI and a ring of 18 IsiA copies, see Fig. 1. b, c Monomeric PSI with rings of 14 and 21 IsiA copies, respectively. The difference in detail between the two rings is related to the alignment procedure, see text. d-e Monomeric PSI complexes associated with an incomplete inner ring and outer ring. The inner ring is composed of six IsiA copies in register. f Monomeric PSI complex with a flexible attachment of incomplete inner and outer rings with a larger number of IsiA copies. Space bar for all frames equals $100 \AA$ 

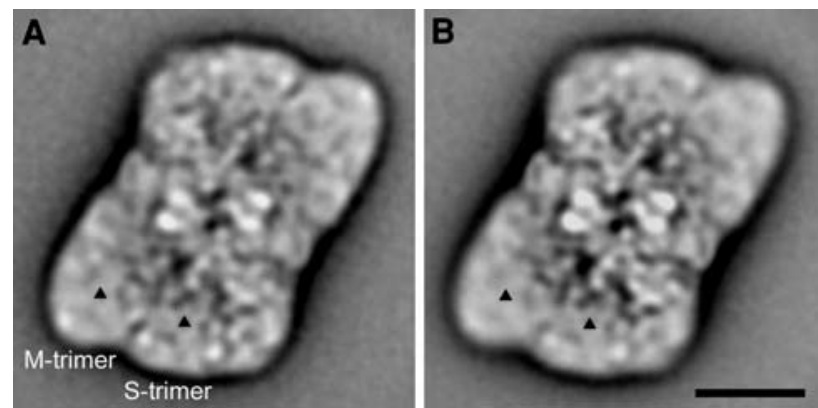

Fig. 5 Analysis of the $\mathrm{C}_{2} \mathrm{~S}_{2} \mathrm{M}_{2}$ supercomplex of photosystem II. a A projection map at about $13 \AA$ shows the exact positions of S-trimers and M-trimer of the LHCII; the triangles indicate the position of the threefold symmetry axis in the center of the trimer. b A projection map, focused on improving the centre of the supercomplex plus the Strimer region. In this map, these areas have been slightly sharpened, but at the cost of the M-trimer. Note: no symmetry was imposed during or after the analysis. Space bar equals $100 \AA$

rings. The fact that the outer ring has seven more copies of IsiA than the inner ring explains why it becomes overall better aligned in Fig. 4b. Further analysis showed that the rotational flexibility between both rings appeared to be about $2-3^{\circ}$, on the average.

Supercomplexes with incomplete rings also show a variable flexibity. The best complexes have an inner ring of six copies (1/3 of the complete ring around a trimer) and 67 copies in the outer ring (Fig. 4d, e). The particles with larger numbers of copies look more fuzzy, which reflects a flexible binding between the rings (4F).

In our studies, several other examples of floppy proteins were notified, such as the $\mathrm{C}_{2} \mathrm{~S}_{2} \mathrm{M}_{2}$ supercomplex of photosystem II, which is composed of a dimeric $\mathrm{C}_{2}$ core and two LHCII S-trimers and M-trimers (Dekker and Boekema 2005). A current projection map at about $13 \AA$ resolution shows that the M-trimer is less well fixed in position than the S-trimer (R. Kouriil, unpublished data). The projection map of Fig. 5a was obtained by improving the complete structure. If one tries to sharpen part of the structure, such as the S-trimer region, by masking out the M-trimer in a reference image for alignments, this leads to a loss in resolution at this particular part (Fig. 5b), clearly indicating that the structure is not rigid at all.

\section{Examples of single particle EM: analysis without purification steps}

Isolated photosynthetic membranes can be solubilized and the complete set of proteins can be used for EM. After single particle analysis, all the (larger) membrane protein projections can be sorted and averaged, as for example with solubilized cyanobacterial membranes (Fig. 6). Some of the obtained projections can be easily assigned, because
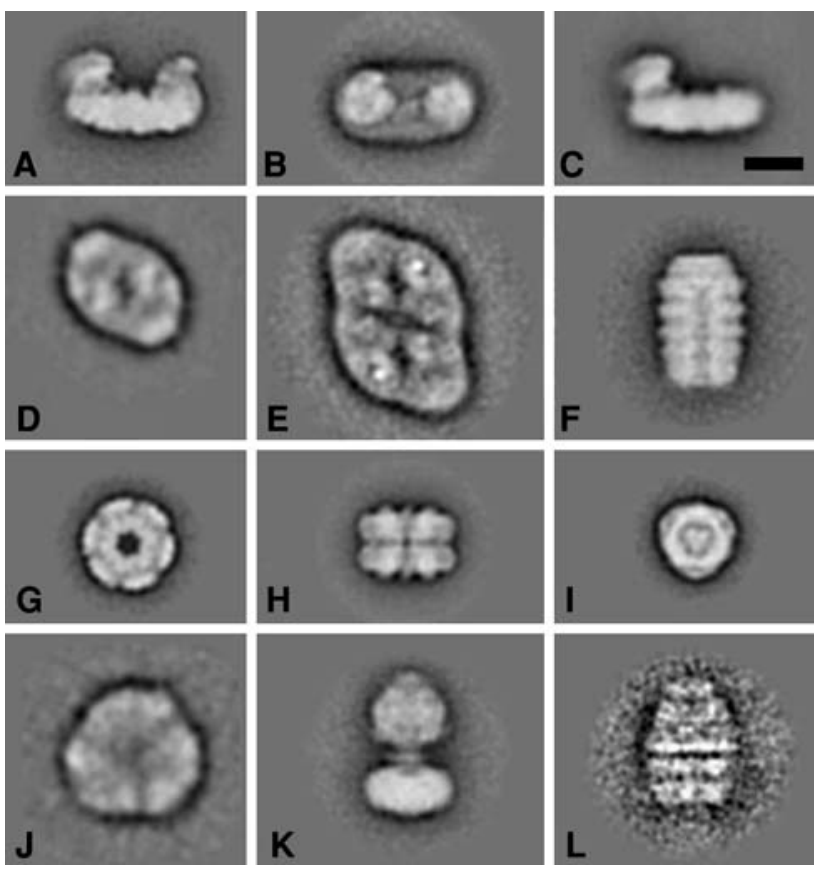

Fig. 6 Exploring transient membrane complexes by applying single particle EM without purification steps. A gallery of $2 \mathrm{D}$ projection maps of solubilized membrane complexes from the cyanobacteria Thermosynechoccus elongatus and Synechocystis PCC 6803. a NDH1 side view from $T$. elongatus $\mathbf{b}$ NDH-1 top view from T. elongatus. c Purified NDH-1 from Synechocystis (reproduced from Arteni et al. 2006). d Photosystem II dimeric complex from Synechocystis. e Photosystem II double dimer complex from Synechocystis. f Rodlike protein complex of unknown origin/function with a variable extension at the base, which could be detergent and lipid, from $T$. elongatus. $\mathbf{g}$, h A water-soluble hexagonal particle, tentatively assigned to glutamine synthetase in top- and side-view position, respectively. i Cyanobacterial fragment with trimeric symmetry assigned to allophycocyanin. j Trimeric photosystem I complex. k Proton ATP synthase complex. I Structure assigned to the GroELGroES supercomplex. Space bar for all frames equals $100 \AA$

structures have been solved. Well-known protein complexes such as trimeric photosystem I (PSI) (Fig. 6j), dimeric photosystem II (Fig. 6d), and the ATP synthase (Fig. 6k) are recognizable from their shape and size. There are, however, also complexes of unknown composition such as a novel "rod-like" particle (Fig. 6f) that could have to do with phycobilisomes. The averaged projections of the frames Fig. 6a, b can be assigned to side- and top-views of the NAD $(\mathrm{P}) \mathrm{H}$ dehydrogenase complex (abbreviated NDH-1 complex). Interestingly, the side-view map of Fig. 6a reveals an U-shaped particle, which has an extra density on its hydrophobic arm, as compared with the classical L-shaped particle obtained by purification (Fig. 6c, Arteni et al. 2006). Apparently, the standard purification procedure of NDH-1, which includes dodecyl maltoside as detergent for solubilization, results in the loss of specific subunits. This observation triggered the assignment of this extra density. Because a purification of the U-shaped NDH-1 
complex was expected to be difficult, a strategy was used to repeat the solubilization and single particle analysis from mutants lacking specific components, expected to be part of NDH-1. From the analysis of the NDH-1 particles from a mutant lacking CupA and a double mutant lacking Cup $\mathrm{A} / \mathrm{B}$, it was proven that the unknown density was CupA, because only L-shaped particles were observed in the mutants (Folea et al. 2008).

This strategy of "no-purification" was also successfully applied to the PSI-LHCII supercomplex of the green plant Arabidopsis thaliana, a transient complex, which is difficult to purify, if at all possible (Kouřil et al. 2005b). It showed that one LHCII trimer is attached on PSI at the side of the $\mathrm{PsaH},-\mathrm{P},-\mathrm{O}$, and $-\mathrm{K}$ subunits.

Acknowledgments This study has been supported by the Council for Chemical Research of the Netherlands Organization for Scientific Research (NWO).

Open Access This article is distributed under the terms of the Creative Commons Attribution Noncommercial License which permits any noncommercial use, distribution, and reproduction in any medium, provided the original author(s) and source are credited.

\section{References}

Adrian M, Dubochet J, Lepault J, McDowall AW (1984) Cryoelectron microscopy of viruses. Nature 308:32-36

Arteni AA, Nowaczyk M, Lax J, Kouřil R, Rögner M, Boekema EJ (2005) Single particle electron microscopy in combination with mass spectrometry to investigate novel complexes of membrane proteins. J Struct Biol 149:325-331

Arteni AA, Zhang P, Battchikova N, Ogawa T, Aro EM, Boekema EJ (2006) Structural characterization of NDH-1 complexes of Thermosynechococcus elongatus by single particle electron microscopy. Biochim Biophys Acta 1757:1469-1475

Bibby TS, Nield J, Barber J (2001) Three-dimensional model and characterization of the iron stress-induced CP43'-Photosystem I supercomplex isolated from the cyanobacterium Synechocystis PCC 6803. J Biol Chem 276:43246-43252

Boekema EJ (1991) Negative staining of integral membrane proteins. Micron Microsc Acta 22:361-369

Boekema EJ, van Heel MG (1989) Molecular shape of Lumbricus terrestris erythrocruorin studied by electron microscopy and image processing. Biochim Biophys Acta 957:370-379

Boekema EJ, Hifney A, Yakushevska AY, Piotrowski M, Keegstra W, Berry S, Michel KP, Pistorius EK, Kruip J (2001) A giant chlorophyll-protein complex induced by iron deficiency in cyanobacteria. Nature 412:745-748

Böttcher B, Gräber P, Boekema EJ (1992) The structure of Photosystem I from the thermophilic cyanobacterium Synechococcus sp. determined by electron microscopy of two-dimensional crystals. Biochim Biophys Acta 1100:125-136

Carragher B, Potter CS, Sigworth FJ (2007) Software tools for macromolecular microscopy. J Struct Biol 57:1-2

De Carlo S, Boisset N, Hoenger A (2008) High-resolution singleparticle 3D analysis on GroEL prepared by cryo-negative staining. Micron 39:934-943
Dekker JP, Boekema EJ (2005) Supermolecular organization of the thylakoid membrane proteins in green plants. Biochim Biophys Acta 1706:12-39

Faruqi AR, Henderson R (2007) Electronic detectors for electron microscopy. Curr Opin Struct Biol 17:549-555

Folea IM, Zhang P, Nowaczyk MM, Ogawa T, Aro EM, Boekema EJ (2008) Single particle analysis of thylakoid proteins from Thermosynechococcus elongatus and Synechocystis 6803: localization of the CupA subunit of NDH-1. FEBS Lett 582:249-254

Frank J (2002) Single-particle imaging of macromolecules by cryoelectron microscopy. Annu Rev Biophys Biomol Struct 31:309319

Golas MM, Sander B, Will CL, Lührmann R, Stark H (2003) Molecular architecture of the multiprotein splicing factor SF3b. Science 300:980-984

Harris JR, Horne RW (1994) Negative staining — a brief assessment of current technical benefits, limitations and future possibilities. Micron 25:5-13

Heinemeyer J, Braun HP, Boekema EJ, Kouřil R (2007) A structural model of the cytochrome $\mathrm{c}$ reductase/oxidase supercomplex from yeast mitochondria. J Biol Chem 282:12240-12248

Henderson R (1995) The potential and limitations of neutrons, electrons and X-rays for atomic resolution microscopy of unstained biological molecules. Q Rev Biophys 28:171-193

Henderson R, Baldwin JM, Ceska TA, Zemlin F, Beckmann E, Downing KH (1990) Model for the structure of bacteriorhodopsin based on high-resolution electron cryo-microscopy. J Mol Biol 213:899-920

Kouřil R, Arteni AA, Lax J, Yeremenko N, D’Haene S, Rögner M, Matthijs HCP, Dekker JP, Boekema EJ (2005a) Structure and functional role of supercomplexes of IsiA and Photosystem I in cyanobacterial photosynthesis. FEBS Lett 579:3253-3257

Kouřil R, Zygadlo A, Arteni A, de Wit CD, Dekker JP, Jensen PE, Scheller HV, Boekema EJ (2005b) Structural characterization of a complex of photosystem I and light-harvesting complex II of Arabidopsis thaliana. Biochemistry 44:10935-10940

Kühlbrandt W, Wang DN, Fujiyoshi Y (1994) Atomic model of plant light-harvesting complex by electron crystallography. Nature 367:614-621

Ludtke SJ, Matthew L, Baker L, Chen DH, Song JL, Chuang DT, Chiu W (2008) De Novo backbone trace of GroEL from single particle electron cryomicroscopy. Structure 16:441-448

Massower WH, Lai PF, Marsh P (2001) Negative staining permits $4.0 \AA$ resolution with low-dose electron diffraction of catalase crystals. Ultramicroscopy 90:7-12

Mitra K, Frank J (2006) Ribosome dynamics: insights from atomic structure modeling into cryo-electron microscopy maps. Annu Rev Biophys Biomol Struct 35:299-317

Royer WE, Sharma H, Strand K, Knapp JE, Bhyravbhatla B (2006) Lumbricus erythrocruorin at $3.5 \AA$ resolution: architecture of a megadalton respiratory complex. Structure 14:1167-1177

Stahlberg H, Walz T (2008) Molecular electron microscopy: state of the art and current challenges. ACS Chem Biol 3:268-281

Stark H, Dube P, Lührmann R, Kastner B (2001) Arrangement of RNA and proteins in the spliceosomal particle. Nature 409:539542

Unger V (2001) Electron cryomicroscopy methods. Curr Opin Struct Biol 11:548-554

Van Heel M, Gowen B, Matadeen R, Orlova EV, Finn R, Pape T, Cohen D, Stark H, Schmidt R, Schatz M, Patwardhan A (2000) Single-particle electron cryo-microscopy: towards atomic resolution. Q Rev Biophys 33:307-369

Yamaguchi M, Danev R, Nishiyama K, Sugawara K, Nagayama K (2008) Zerike phase contrast electron microscopy of iceembedded influenza A virus. Ultramicroscopy 162:271-276 
Yeager M, Unger VM, Mitra AK (1999) Three-dimensional structure of membrane proteins determined by two-dimensional crystallization, electron cryomicropscopy, and image analysis. Methods Enzymol 294:135-180

Yeremenko N, Kouřil R, Ihalainen JA, D'Haene S, van Oosterwijk N, Andrizhiyevskaya EG, Keegstra W, Dekker HL, Hagemann M, Boekema EJ, Matthijs HCP, Dekker JP (2004) Supramolecular organization and dual function of the IsiA chlorophyll-binding protein in cyanobacteria. Biochemistry 43:10308-10313

Zhang X, Settembre E, Xu C, Dormitzer PR, Bellamy R, Harrison SC, Grigorieff N (2008) Near-atomic resolution using electron cryomicroscopy and single-particle reconstruction. Proc Natl Acad Sci USA 105:1867-1872 